\title{
Preoperative albumin-to-fibrinogen ratio predicts chemotherapy resistance and prognosis in patients with advanced epithelial ovarian cancer
}

\author{
Wen Yu, Zhongxue Ye, Xi Fang, Xingzhi Jiang and Yafen Jiang*
}

\begin{abstract}
Background: Epithelial ovarian cancer (EOC) is the majority ovarian cancer (OC) type with a poor prognosis. This present study aimed to investigate potential prognostic factors including albumin-to-fibrinogen ratio (AFR) for advanced EOC patients with neoadjuvant chemotherapy (NAC) followed by debulking surgery.

Methods: A total of 313 advanced EOC patients with NAC followed by debulking surgery from 2010 to 2017 were enrolled. The predictive value of AFR for the overall survival (OS) was evaluated by receiver operating characteristic (ROC) curve analysis. The univariate and multivariate Cox proportional hazards regression analyses were applied to investigate prognostic factors for advanced EOC patients. The association between preoperative AFR and progression free survival (PFS) or OS was determined via the Kaplan-Meier method using log-rank test.

Results: The ROC curve analysis showed that the cutoff value of preoperative AFR in predicting OS was determined to be 7.78 with an area under the curve (AUC) of $0.773(P<0.001)$. Chemotherapy resistance, preoperative CA125 and AFR were independent risk factors for PFS in advanced EOC patients. Furthermore, chemotherapy resistance, residual tumor and AFR were significant risk factors for OS by multivariate Cox analysis. A low preoperative AFR ( $\leq 7.78)$ was significantly associated with a worse PFS and OS via the Kaplan-Meier method by log-rank test $(P<0.001)$.

Conclusions: A low preoperative AFR was an independent risk factor for PFS and OS in advanced EOC patients with NAC followed by debulking surgery.
\end{abstract}

Keywords: Epithelial ovarian cancer, Prognosis, chemotherapy resistance, Albumin-to-fibrinogen ratio

\section{Introduction}

Epithelial ovarian cancer (EOC), as the majority ovarian cancer (OC) type, represents the most lethal gynecologic malignancy [1] and the 5th leading cause of cancerrelated death worldwide [2]. Despite the improved therapeutic strategies, OC is still a deadly disease with a mortality rate over 30\% [3]. As reported by previous studies, the median progression-free survival (PFS) is only $16-22$ months and the 5 -year overall survival (OS) rate is less than $30 \%$ [4]. Unfortunately, most EOC cases

\footnotetext{
* Correspondence: jiangyafennb@sina.com

Wen $\mathrm{Yu}$ is the first author

Department of Gynecology, HwaMei Hospital, University Of Chinese
Academy Of Sciences, NO 41, Xibei Street, Ningbo City 315000, Zhejiang

Department of Gynecology, HwaMei Hospital, University Of Chinese
Academy Of Sciences, NO 41, Xibei Street, Ningbo City 315000, Zhejiang Province, China
}

were diagnosed at advanced stages by reason of its asymptomatic rapid progression. Over the last decades, the primary standard treatment involves cytoreductive surgery and platinum/taxane based chemotherapy [5]. However, the the overall survival of EOC remains poor due to the advanced stage at diagnosis, chemoresistance and high tumor recurrence rate [6]. Therefore, to investigate potential predictors is urgently needed for the improvement of diagnosis, new therapeutic target, disease activity and treatment response monitoring.

Recently, a great deal of evidence has shown that the status of systemic inflammatory response to tumor and nutrition closely correlates with the prognosis in various cancers [7, 8]. Albumin (Alb), which has been commonly used to for the nutritional and inflammatory status 
assessment, is indicated as a potential prognostic factor for advanced cancer [9]. Increasing evidence has also revealed the close association between coagulation and tumor progression and metastasis [10, 11]. Fibrinogen (Fib), which is an essential protein in the coagulation cascade and an acute-phase reaction protein for systemic inflammation like C-reactive protein (CRP), plays an important role in tumor development [12]. Albumin-tofibrinogen ratio (AFR), which takes both Alb and Fib into account, has been indicated as a prognostic factor for various malignancies including non-small cell lung cancer (NSCLC) [13], chronic lymphocytic leukemia [14], and breast cancer [15], etc. However, no study has investigated the association between AFR and EOC until now. Hence, the aim of this study was to investigate whether circulating AFR could serve an effective prognostic biomarker for EOC.

\section{Material and methods \\ Patients}

This retrospective study was approved by the Medical Institutional Ethics Committee of Hwa Mei Hospital and Zhejiang province. Eligible subjects with newly diagnosed EOC admitted at the Department of Gynecology, Hwa Mei Hospital, University Of Chinese Academy Of Sciences from 2010 to 2017 were enrolled into this study population. The inclusion criteria were as follows: (a) newly histologically diagnosed advanced EOC patients with the International Federation of Gynecology and Obstetrics (FIGO) stage III or IV; (b) available preoperative serum expressions of Alb and Fig; (c) patients who underwent neoadjuvant chemotherapy (NAC) followed by debulking surgery; (c) patients with adequate clinicopathological data and (d) patients with a follow up for at least 12 months. The exclusion criteria were as follows: (a) patients who had clinical evidence of infection or inflammatory conditions; (b) patients who were with other malignancies; (c) patients who were with autoimmune or hematological diseases, abnormal liver or renal function and (d) patients who were lost to follow-up or without complete data. Informed consent was obtained from all the enrolled patients who underwent cytoreduction and chemotherapy.

\section{Treatment and data collection}

A same experienced pathologist who was blinded to this study was invited to review all the surgical specimens and make the pathological evaluation. All the included patients underwent three courses of adjuvant first-line platinum-based chemotherapy before the primary cytoreduction surgery. Each enrolled patient was required to perform the examinations of whole body computed tomography (CT) or positron emission tomography/ computed tomography (PET/CT). The tumor stage was determined for all patients following the guidance FIGO system. Demographics, clinicpathological parameters and preoperative laboratory tests were collected from medical records. Preoperative fasting peripheral blood samples were obtained on 1 day prior to operation and were then processed within $48 \mathrm{~h}$ for laboratory variable detections. The blood cells, C-reactive protein (CRP), Alb, Fib and CA125 were detected using the obtained blood samples. The AFR was calculated by Alb (g/L)/Fib $(\mathrm{g} / \mathrm{L})$ ratio.

\section{Prognosis definition}

At each visit during follow up, clinical, imaging examinations and the serum CA-125 expression were assessed for treatment evaluation. The primary end point of this study was PFS, overall survival (OS), or the last follow

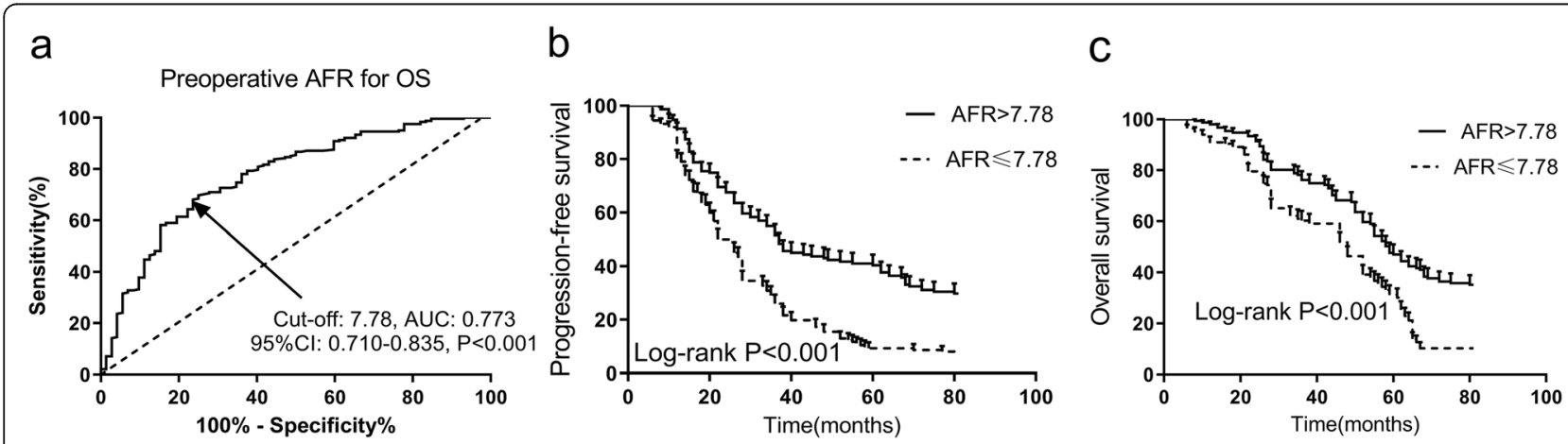

Fig. 1 AFR and prognosis in advanced EOC patients. a Predictive value of preoperative AFR for OS in advanced EOC by ROC curve. The cutoff value of AFR in predicting OS was determined to be 7.78 with an AUC of $0.773,95 \% \mathrm{Cl}$ of $0.710-0.835$, a sensitivity of $64.32 \%$ and a specificity of $77.78 \%$, respectively $(P<0.001)$; (b) PFS in advanced EOC patients according to AFR by Kaplan-Meier curve analysis. Patients with a lower preoperative AFR ( $\leq 7.78)$ had a worse PFS than those with a high preoperative AFR $(>7.78)$ by log-rank test $(P<0.001)$; (c) OS in advanced EOC patients according to AFR by Kaplan-Meier curve analysis. Patients with a lower preoperative AFR ( $\leq 7.78)$ had a worse OS than those with a high preoperative AFR $(>7.78)$ by log-rank test $(P<0.001)$. AFR, albumin-to-fibrinogen ratio; PFS, progression-free survival; OS; overall survival; EOC, epithelial ovarian cancer; $\mathrm{ROC}$, receiver operating characteristic; $\mathrm{AUC}$, the area under the curve; $\mathrm{Cl}$, confidence interval 
Table 1 Clinicpathological parameters associated with AFR in advanced EOC patients

\begin{tabular}{|c|c|c|c|}
\hline \multirow[b]{2}{*}{ Parameters } & \multicolumn{2}{|l|}{ AFR } & \multirow[b]{2}{*}{$p$ value } \\
\hline & Low $(\leq 7.78)$ & High $(>7.78)$ & \\
\hline Number & 151 & 162 & - \\
\hline Age (years) & $65.8 \pm 8.7$ & $63.1 \pm 7.8$ & $0.004^{*}$ \\
\hline BMI $\left(\mathrm{kg} / \mathrm{m}^{2}\right)$ & $23.1 \pm 2.3$ & $23.4 \pm 2.1$ & 0.229 \\
\hline Current smokers (n, \%) & $18(11.9)$ & $24(14.8)$ & 0.453 \\
\hline Alcohol (n, \%) & $14(9.3)$ & $19(11.7)$ & 0.479 \\
\hline Comorbidities (n, \%) & & & - \\
\hline Diabetes & $22(14.6)$ & $17(10.5)$ & 0.275 \\
\hline Hypertension & $27(17.9)$ & $22(13.6)$ & 0.295 \\
\hline Hyperlipemia & $15(9.9)$ & $12(7.4)$ & 0.426 \\
\hline Histological grade $(\mathrm{n}, \%)$ & & & $0.042^{*}$ \\
\hline G1 & $76(50.3)$ & $100(61.7)$ & - \\
\hline $\mathrm{G} 2 / 3$ & $75(49.7)$ & $62(38.3)$ & - \\
\hline Histological type (n, \%) & & & 0.444 \\
\hline Serous & $106(70.2)$ & $120(74.1)$ & - \\
\hline Non-serous & $45(29.8)$ & $42(25.9)$ & - \\
\hline FIGO stage $(n, \%)$ & & & $0.032^{*}$ \\
\hline III & $113(74.8)$ & $137(84.6)$ & - \\
\hline IV & $38(25.2)$ & $25(15.4)$ & - \\
\hline ECOG PS $(n, \%)$ & & & $0.016^{*}$ \\
\hline 0 & $101(66.9)$ & $128(79.0)$ & - \\
\hline$\geq 1$ & $50(33.1)$ & $34(21.0)$ & - \\
\hline Chemotherapy resistance $(n, \%)$ & $54(35.8)$ & $40(24.7)$ & $0.033^{*}$ \\
\hline Residual tumor (cm) & & & $0.005^{*}$ \\
\hline$\leq 1$ & $100(66.2)$ & $130(80.2)$ & - \\
\hline$>1$ & $51(33.8)$ & $32(19.8)$ & - \\
\hline
\end{tabular}

EOC, Epithelial ovarian cancer; BMI, Body mass index; FIGO, International Federation of Gynecology and Obstetrics; AFR, Albumin-to-fibrinogen ratio; ECOG PS, Eastern cooperative oncology group performance status. $P$-values were calculated by Student's $t$ test, Mann-Whitney $U$ test or Chi-squared test. * $P<0.05$.

up (December, 2017). The chemotherapy responses was assessed following the guidance of the Response Evaluation Criteria in Solid Tumors (RECIST) [16]. Chemotherapy sensitivity was defined as complete response (CR) or partial response (PR), whereas chemotherapy resistance was defined as stable disease (SD) or progressive disease (PD). PFS was defined as the duration from the date of initial surgery to the evidence of tumor progression, death or follow up deadline. OS was defined as the duration from the date of initial surgery to death or the last follow-up.

\section{Statistical analysis}

Statistical analyses were performed with the software of GraphPad prism (version. 5.0, GraphPad Inc., San Diego, CA, USA) and SPSS (version 19.0, SPSS Inc., Chicago,
Table 2 Laboratory tests associated with AFR in advanced EOC patients

\begin{tabular}{|c|c|c|c|}
\hline \multirow[b]{2}{*}{ Laboratory tests } & \multicolumn{2}{|l|}{ AFR } & \multirow[b]{2}{*}{$p$ value } \\
\hline & Low $(\leq 7.78)$ & High $(>7.78)$ & \\
\hline Hemoglobin (g/L) & $102.4 \pm 11.5$ & $104.1 \pm 12.5$ & 0.212 \\
\hline Platelet $\left(10^{9} / \mathrm{L}\right)$ & $164.4 \pm 35.8$ & $170.7 \pm 41.3$ & 0.152 \\
\hline $\operatorname{WBC}\left(10^{9} / \mathrm{L}\right)$ & $7.0 \pm 1.9$ & $7.2 \pm 2.2$ & 0.392 \\
\hline Albumin (g/L) & $38.9 \pm 4.6$ & $39.2 \pm 3.9$ & 0.533 \\
\hline Total protein (g/L) & $70.1 \pm 7.8$ & $69.6 \pm 6.5$ & 0.537 \\
\hline Total cholesterol (mmol/L) & $4.3 \pm 0.6$ & $4.2 \pm 0.7$ & 0.177 \\
\hline Triglyceride (mmol/L) & $1.2 \pm 0.5$ & $1.3 \pm 0.5$ & 0.078 \\
\hline CRP (ng/L) & $7.4 \pm 10.1$ & $5.3 \pm 7.1$ & $0.033^{*}$ \\
\hline CA125 (U/mL) & $145.1 \pm 189.3$ & $87.2 \pm 128.1$ & $0.002^{*}$ \\
\hline
\end{tabular}

IL, USA). Data are presented with number with proportion, or mean \pm standard deviation (SD). The predictive value of AFR for the OS as well as the cut-off value was evaluated by receiver operating characteristic (ROC) curve analysis and the Youden index. The comparisons between categorical variables were performed with the Chi-square test, while continuous variables with Student $\mathrm{t}$ test or Mann-Whitney $\mathrm{U}$ test as appropriate. The univariate and multivariate analyses were performed to evaluate the prognostic factors using the Cox proportional hazard model and only those factors with a $p$ value $<0.1$ in the univariate mode were further analyzed in the multivariate mode. OS and PFS were calculated using Kaplan-Meier method and the log-rank test. A two-sided $P<0.05$ was accepted as statistically different.

\section{Results}

\section{Patient characteristics}

A total of 375 subjects were primarily enrolled according to the inclusion criteria and 62 were excluded due to the exclusion criteria (10 had infection or inflammatory conditions, 12 were with other malignancies, 13 were with autoimmune or hematological diseases, 20 were with abnormal liver or renal function and 27 were lost to follow-up or without complete data). Finally, 313 advanced EOC patients with a mean age of 64.4 years were included the analysis. As shown in Fig. 1a, the ROC curve analysis showed that the cutoff value of AFR in predicting OS was determined to be 7.78 with an area under the curve (AUC) of $0.773,95 \%$ CI of $0.710-0.835$, a sensitivity of $64.32 \%$ and a specificity of $77.78 \%$, respectively $(P<0.001)$. According to the baseline cutoff value, all the 313 were categorized into two groups, 151 $(48.2 \%)$ in the low-AFR group (AFR $\leq 7.78)$ and 162 $(51.8 \%)$ in the high-AFR group (AFR $>3.45)$. The 
Table 3 Risk factors for PFS by univariate and multiple Cox regression analysis

\begin{tabular}{|c|c|c|c|c|}
\hline & Univariate & & Multivariate & \\
\hline Parameters & $\mathrm{HR}(95 \% \mathrm{Cl})$ & $p$ value & $\mathrm{HR}(95 \% \mathrm{Cl})$ & $p$ value \\
\hline Age (high vs low) & $1.50(1.11-2.08)$ & $0.016^{*}$ & $1.17(0.85-1.62)$ & 0.301 \\
\hline BMI (high vs low) & $0.82(0.61-1.15)$ & 0.251 & & \\
\hline Current smokers (yes vs no) & $1.28(0.97-1.70)$ & 0.112 & & \\
\hline Alcohol (yes vs no) & $1.16(0.89-1.53)$ & 0.247 & & \\
\hline Diabetes (yes vs no) & $1.03(0.75-1.41)$ & 0.822 & & \\
\hline Hypertension (yes vs no) & $1.06(0.73-1.49)$ & 0.794 & & \\
\hline Hyperlipemia (yes vs no) & $0.85(0.42-1.65)$ & 0.654 & & \\
\hline Histological grade (G2/3 vs G1) & $1.76(1.19-2.63)$ & $0.012^{*}$ & $1.63(0.92-2.77)$ & 0.093 \\
\hline Histological type (serous vs non-serous) & $1.21(0.89-1.59)$ & 0.179 & & \\
\hline FIGO stage (IV vs III) & $1.72(1.09-2.73)$ & $0.024^{*}$ & $1.44(0.79-2.69) 0.254$ & \\
\hline ECOG PS ( $\geq 1$ vs 0$)$ & $0.86(0.71-1.07)$ & 0.852 & & \\
\hline Chemotherapy resistance (yes vs no) & $2.07(1.35-3.17)$ & $0.011^{*}$ & $1.84(1.12-2.88)$ & $0.032^{*}$ \\
\hline Residual tumor (> $1 \mathrm{~cm}$ vs $\leq 1 \mathrm{~cm}$ ) & $2.01(0.94-4.31)$ & 0.071 & $1.61(0.91-2.85)$ & 0.121 \\
\hline Hemoglobin (high vs low) & $1.84(0.69-4.78)$ & 0.211 & & \\
\hline Platelet (high vs low) & $1.55(0.38-5.78)$ & 0.529 & & \\
\hline WBC (high vs low) & $1.86(0.86-3.89)$ & 0.127 & & \\
\hline Albumin (high vs low) & $0.92(0.57-1.42)$ & 0.721 & & \\
\hline Total protein (high vs low) & $0.81(0.44-1.48)$ & 0.472 & & \\
\hline Total cholesterol (high vs low) & $0.75(0.47-1.24)$ & 0.261 & & \\
\hline Triglyceride (high vs low) & $1.26(0.70-2.22)$ & 0.425 & & \\
\hline CA125 (high vs low) & $1.72(1.22-2.44)$ & $0.011^{*}$ & $1.42(1.02-2.05)$ & $0.042^{*}$ \\
\hline CRP (high vs low) & $1.36(0.69-2.64)$ & 0.373 & & \\
\hline $\operatorname{AFR}(\leq 7.78$ vs $>7.78)$ & $1.56(1.21-2.05)$ & $0.007^{*}$ & $1.38(1.04-1.82)$ & $0.019^{*}$ \\
\hline
\end{tabular}

EOC, Epithelial ovarian cancer; PFS, Progression-free survival; BMI, Body mass index; FIGO, International Federation of Gynecology and Obstetrics; AFR, Albumin-tofibrinogen ratio; ECOG PS, Eastern cooperative oncology group performance status; $W B C$, White blood cell; $C R P$, C-reactive protein; $H R$, Hazard ratio; $C l$, Confidence interval. ${ }^{*} P<0.05$.

baseline demographic and clinicopathological characteristics associated with AFR are detailed exhibited in Table 1. In comparison with the low-AFR group, patients in the high-AFR group showed a lower age $(P=$ $0.004)$, ECOG PS $(P=0.016)$, histological grade $(P=$ $0.042)$ and FIGO stage $(P=0.032)$. High AFR expression was significantly associated with an increased incidence of first-line platinum-based chemotherapy resistance $(P=0.033)$. Patients with preoperative AFR $\leq 7.78$ had a higher prevalence of postoperative residual tumor larger than $1 \mathrm{~cm}(P=0.005)$. However, no statistically differences were noted between the high-AFR and low-AFR groups in respect to BMI, smoking and alcohol habits, preoperative comorbidities and histological type $(P>$ $0.05)$. Other laboratory tests including blood cells analyses, CRP and CA125 were also analyzed. As shown in Table 2, the results indicated that high AFR expression was significantly associated with lower expressions of CRP $(P=0.033)$ and CA125 $(P=0.002)$. No significant differences were observed in blood cell analyses between low-AFR and high-AFR groups $(P>0.05)$.

\section{Risk factors for PFS and OS in advanced EOC patients}

As shown in Table 3, potential risk factors for PFS in advanced EOC patients were assessed by univariate and multivariate Cox proportional regression analyses. Potential risk factors with a $P$ value $<0.1$ by the univariate Cox analysis including Age, histological grade, FIGO stage, chemotherapy resistance, residual tumor, CA125 and AFR were enrolled into the multivariate Cox analysis. The results indicated that chemotherapy resistance (HR: 1.84, 95 CI\%: $1.12-2.88, P=0.032$ ), CA125 (HR: 1.42, 95 CI\%: $1.02-2.05, P=0.042)$ and AFR (HR: 1.38 , 95 CI\%: 1.04-1.82, $P=0.019)$ were independent risk factors for PFS in advanced EOC patients. Furthermore, chemotherapy resistance (HR: 1.30, 95 CI\%: 1.01-1.67, $P=0.032$ ), residual tumor (HR: $1.48,95 \mathrm{CI} \%$ : $1.01-2.21$, $P=0.046)$ and AFR (HR: 2.19, 95 CI\%: $1.18-3.67 P=$ 
Table 4 Risk factors for OS by univariate and multiple Cox analysis

\begin{tabular}{|c|c|c|c|c|}
\hline \multirow[b]{2}{*}{ Parameters } & \multicolumn{2}{|l|}{ Univariate } & \multicolumn{2}{|l|}{ Multivariate } \\
\hline & $\mathrm{HR}(95 \% \mathrm{Cl})$ & $p$ value & $\mathrm{HR}(95 \% \mathrm{Cl})$ & $p$ value \\
\hline Age (high vs low) & $1.23(0.84-1.81)$ & 0.304 & & \\
\hline BMI (high vs low) & $0.53(0.24-1.15)$ & 0.112 & & \\
\hline Current smokers (yes vs no) & $1.09(0.76-1.57)$ & 0.647 & & \\
\hline Alcohol (yes vs no) & $1.17(0.82-1.67)$ & 0.384 & & \\
\hline Diabetes (yes vs no) & $1.24(0.71-2.13)$ & 0.435 & & \\
\hline Hypertension (yes vs no) & $1.31(0.83-2.05)$ & 0.247 & & \\
\hline Hyperlipemia (yes vs no) & $0.85(0.54-1.33)$ & 0.468 & & \\
\hline Histological grade (G2/3 vs G1) & $1.57(1.03-2.41)$ & $0.034^{*}$ & $0.92(0.81-1.22)$ & 0.068 \\
\hline Histological type (serous vs non-serous) & $0.49(0.83-2.64)$ & 0.181 & & \\
\hline FIGO stage (IV vs III) & $1.89(1.17-3.11)$ & $0.014^{*}$ & $1.58(0.77-3.35)$ & 0.221 \\
\hline ECOG PS ( $\geq 1$ vs 0 ) & $1.12(0.74-1.69)$ & 0.603 & & \\
\hline Chemotherapy resistance (yes vs no) & $1.27(1.03-1.62)$ & $0.021^{*}$ & $1.30(1.01-1.67)$ & $0.032^{*}$ \\
\hline Residual tumor (> $1 \mathrm{~cm}$ vs $\leq 1 \mathrm{~cm}$ ) & $1.32(1.04-1.68)$ & $0.022^{*}$ & $1.48(1.01-2.21)$ & $0.046^{*}$ \\
\hline Hemoglobin (high vs low) & $0.78(0.39-1.56)$ & 0.478 & & \\
\hline Platelet (high vs low) & $1.55(0.75-3.18)$ & 0.237 & & \\
\hline WBC (high vs low) & $1.39(0.77-2.51)$ & 0.278 & & \\
\hline Albumin (high vs low) & $0.65(0.38-1.12)$ & 0.112 & & \\
\hline Total protein (high vs low) & $0.89(0.55-1.45)$ & 0.642 & & \\
\hline Total cholesterol (high vs low) & $0.69(0.31-1.52)$ & 0.359 & & \\
\hline Triglyceride (high vs low) & $1.50(0.83-2.73)$ & 0.181 & & \\
\hline CA125 (high vs low) & $1.41(0.73-2.73)$ & 0.304 & & \\
\hline CRP (high vs low) & $1.17(0.64-2.11)$ & 0.613 & & \\
\hline $\operatorname{AFR}(\leq 7.78$ vs $>7.78)$ & $2.50(1.44-4.09)$ & $0.007^{*}$ & $2.19(1.18-3.67)$ & $0.021^{*}$ \\
\hline
\end{tabular}

EOC, Epithelial ovarian cancer; OS, Orall survival; BMI, Body mass index; FIGO, International Federation of Gynecology and Obstetrics; AFR, Albumin-to-fibrinogen ratio; ECOG PS, Eastern cooperative oncology group performance status; WBC, White blood cell; CRP, C-reactive protein; HR, Hazard ratio; $C l$, Confidence interval. $* P<0.05$.

0.021) were significant risk factors for OS in advanced EOC patients by multivariate Cox analysis (Table 4).

\section{PFS and OS associated with AFR}

To further analyze the prognostic values of preoperative AFR in advanced EOC patients, the Kaplan-Meier curves of PFS and OS are then performed. As shown in Fig. 1b and $\mathrm{c}$, a low preoperative AFR $(\leq 7.78)$ was significantly associated with a worse PFS and OS by log-rank test $(P<0.001)$.

\section{Discussion}

In this present retrospective study, lower AFR was found to be significantly correlated with elder age, higher histological grade, FIGO stage, ECOG PS, larger residual tumor and higher incidence of chemotherapy resistance after cytoreduction surgery in advanced EOC. Furthermore, low preoperative AFR level and chemotherapy resistance were shown to be two independent risk factors associated with a worse PFS and OS based on the multivariate Cox regression model.

To the best of our knowledge, no paper regarding AFR and prognosis in advanced EOC patients has been published until now. This study is the first to suggest AFR as a prognostic factor in advanced EOC patients who underwent neoadjuvant chemotherapy (NAC) followed by debulking surgery. Previously published data in patients with high grade serous ovarian cancer (HGSOC) has indicated that clinical resistance to first-line chemotherapy is significantly associated with a shorter PFS and OS [17]. Our results were in accordance with their conclusions, reflecting the close correlation between chemotherapy resistance and prognosis. Our results has also indicated preoperative AFR as an independent prognostic factor for advanced EOC patients. Previous studies conducted in non-small cell lung cancer individuals have suggested circulating AFR as an effective biomarker to predict prognosis [18], which is in support 
of our results. Preoperative FAR was a strong independent prognostic factor in breast cancer.

A great deal of evidence has reported that the nutritional and systemic inflammatory status were closely associated with prognosis in patients with cancers $[7,8]$. Furthermore, nutrition and inflammation are closely regulated with each other instead of being independent from each other [19]. As an important indicator of nutrition and inflammatory state, the low expression of serum Alb is reported to be significantly associated with poor prognosis in various malignancies including prostate cancer [20], lung cancer [21], breast cancer [15] and chronic lymphocytic leukemia [22]. The close correlation between inflammation and tumorigenesis may be the main possible explanation for the prognostic role of serum Alb in various malignancies [15]. Elevated serum Fib has also been indicated as a predictor for poor prognosis in esophageal cancer [23], breast cancer [24] and EOC [25]. Increased plasma fib expression contributes to elevated blood coagulability state and promotes the intravasation, adhesion and survival of tumor cells, which results in increased metastatic potential in cancer model [26]. Elevated serum Fib and decreased serum Alb have been widely accepted as useful biomarkers for elevated systemic inflammation [19, 27]. Furthermore, AFR, a ratio of Alb to Fib, could amplify the sensitivity of inflammation and nutrition status in EOC patients and it was superior to predict prognosis of EOC in comparison with single Alb or Fib.

Although this was the first study to explore the prognostic role of AFR in advanced EOC patients, it had several limitations. First, this was a retrospective, singlecenter study with relatively small sample size. Second, our conclusions were not validated yet and further study is needed. Third, the AFR expressions were only assessed before the operation, whether postoperative AFR could also serve as a prognostic factor remains unclear. The last, the involved mechanisms for the prognostic role of preoperative AFR for advanced EOC patients were not verified.

\section{Conclusions}

In conclusion, our results indicated that preoperative AFR could serve as a potential predictor for the prognosis in advanced EOC patients with NAC followed by debulking surgery. However, a prospective multiplecentral cohort with a larger sample size are warranted to validate the clinical application of AFR in advanced EOC.

\section{Abbreviations}

AFR: Albumin-to-fibrinogen ratio; AUC: Area under the curve; BMI: Body mass index; Cl: Confidence interval; CRP: C-reactive protein; ECOG PS: Eastern cooperative oncology group performance status; EOC: Epithelial ovarian cancer; FIGO: International Federation of Gynecology and Obstetrics;
HGSOC: High grade serous ovarian cancer; HR: Hazard ratio; NAC: Neoadjuvant chemotherapy; OC: Ovarian cancer; OS: Overall survival; PFS: Progression-free survival; ROC: Receiver operating characteristic; WBC: White blood cell

\section{Acknowledgements}

None.

\section{Authors' contributions}

WY: Project development, Data collection, Data analysis, Manuscript writing. ZY: Data collection. XF: Data analysis and data collection. XJ: Project development. YJ: Project development and data analysis. All authors read and approved the final manuscript.

\section{Funding}

This study was supported by Reasearch Foundation of HwaMei Hospital, University of Chinese Academy of Sciences, China (Grant No. 2019HMKY11)

Availability of data and materials

Please contact the corresponding author Yafen Jiang (jiangyafennb@sina.com).

\section{Ethics approval and consent to participate}

This retrospective study was approved by the Medical Institutional Ethics Committee of Hwa Mei Hospital and Zhejiang province. Informed consent was obtained from all the enrolled patients who underwent cytoreduction and chemotherapy.

Consent for publication

Not applicable.

\section{Competing interests}

None

Received: 26 June 2019 Accepted: 5 September 2019

Published online: 18 September 2019

References

1. Jemal A, Siegel R, Ward E, Hao Y, Xu J, Thun MJ. Cancer statistics, 2009. CA Cancer J Clin. 2009;59(4):225-49.

2. Siegel RL, Miller KD, Jemal A. Cancer statistics, 2015. CA Cancer J Clin. 2015; 65(1):5-29.

3. Jemal A, Bray F, Center MM, Ferlay J, Ward E, Forman D. Global cancer statistics. CA Cancer J Clin. 2011:61(2):69-90.

4. Partridge EE, Barnes MN. Epithelial ovarian cancer: prevention, diagnosis, and treatment. CA Cancer J Clin. 1999;49(5):297-320.

5. Binju M, Padilla MA, Singomat T, Kaur P, Suryo Rahmanto $Y$, Cohen PA, et al. Mechanisms underlying acquired platinum resistance in high grade serous ovarian cancer - a mini review. Biochim Biophys Acta Gen Subj. 2019; 1863(2):371-8.

6. Bast RC Jr, Hennessy B, Mills GB. The biology of ovarian cancer: new opportunities for translation. Nat Rev. Cancer. 2009:9(6):415-28.

7. Gu W, Zhang G, Sun L, Ma Q, Cheng Y, Zhang H, et al. Nutritional screening is strongly associated with overall survival in patients treated with targeted agents for metastatic renal cell carcinoma. J Cachexia Sarcopenia Muscle. 2015;6(3):222-30.

8. Sanchez-Lara K, Turcott JG, Juarez E, Guevara P, Nunez-Valencia C, OnateOcana LF, et al. Association of nutrition parameters including bioelectrical impedance and systemic inflammatory response with quality of life and prognosis in patients with advanced non-small-cell lung cancer: a prospective study. Nutr Cancer. 2012;64(4):526-34.

9. Fiala O, Pesek M, Finek J, Racek J, Minarik M, Benesova L, et al. Serum albumin is a strong predictor of survival in patients with advancedstage non-small cell lung cancer treated with erlotinib. Neoplasma. 2016;63(3):471-6.

10. Ghezzi F, Cromi A, Siesto G, Giudici S, Serati M, Formenti G, et al. Prognostic significance of preoperative plasma fibrinogen in endometrial cancer. Gynecol Oncol. 2010;119(2):309-13.

11. Sun ZQ, Han XN, Wang HJ, Tang Y, Zhao ZL, Qu YL, et al. Prognostic significance of preoperative fibrinogen in patients with colon cancer. World J Gastroenterol. 2014;20(26):8583-91. 
12. Kijima T, Arigami T, Uchikado Y, Uenosono Y, Kita Y, Owaki T, et al. Combined fibrinogen and neutrophil-lymphocyte ratio as a prognostic marker of advanced esophageal squamous cell carcinoma. Cancer Sci. 2017; 108(2):193-9.

13. Chen S, Yan H, Du J, Li J, Shen B, Ying H, et al. Prognostic significance of preresection albumin/fibrinogen ratio in patients with non-small cell lung cancer: A propensity score matching analysis. Clin Chim Acta. 2018;482:203-8.

14. Zou YX, Qiao J, Zhu HY, Lu RN, Xia Y, Cao L, et al. Albumin-to-Fibrinogen Ratio as an Independent Prognostic Parameter in Untreated Chronic Lymphocytic Leukemia: A Retrospective Study of 191 Cases. Cancer Res Treat. 2018.

15. Hwang KT, Chung JK, Roh EY, Kim J, Oh S, Kim YA, et al. Prognostic Influence of Preoperative Fibrinogen to Albumin Ratio for Breast Cancer. J Breast Cancer. 2017;20(3):254-63.

16. Therasse P, Arbuck SG, Eisenhauer EA, Wanders J, Kaplan RS, Rubinstein L, et al. New guidelines to evaluate the response to treatment in solid tumors European Organization for Research and Treatment of Cancer, National Cancer Institute of the United States, National Cancer Institute of Canada. J Natl Cancer Inst. 2000;92(3):205-16.

17. Matte I, Garde-Granger P, Bessette P, Piche A. Serum CA125 and ascites leptin level ratio predicts baseline clinical resistance to first-line platinumbased treatment and poor prognosis in patients with high grade serous ovarian cancer. Am J Cancer Res. 2019;9(1):160-70.

18. Li SQ, Jiang YH, Lin J, Zhang J, Sun F, Gao QF, et al. Albumin-to-fibrinogen ratio as a promising biomarker to predict clinical outcome of non-small cell lung cancer individuals. Cancer Med. 2018;7(4):1221-31.

19. Gupta D, Lis CG. Pretreatment serum albumin as a predictor of cancer survival: a systematic review of the epidemiological literature. Nutr J. 2010;9:69.

20. Wang Y, Chen W, Hu C, Wen X, Pan J, Xu F, et al. Albumin and Fibrinogen Combined Prognostic Grade Predicts Prognosis of Patients with Prostate Cancer. J Cancer. 2017:8(19):3992-4001.

21. Miura K, Hamanaka K, Koizumi T, Kitaguchi Y, Terada Y, Nakamura D, et al. Clinical significance of preoperative serum albumin level for prognosis in surgically resected patients with non-small cell lung cancer: Comparative study of normal lung, emphysema, and pulmonary fibrosis. Lung Cancer. 2017;111:88-95.

22. Wierda WG, O'Brien S, Wang X, Faderl S, Ferrajoli A, Do KA, et al. Prognostic nomogram and index for overall survival in previously untreated patients with chronic lymphocytic leukemia. Blood. 2007;109(11):4679-85.

23. Wakatsuki K, Matsumoto S, Migita K, Ito M, Kunishige T, Nakade H, et al. Preoperative Plasma Fibrinogen is Associated with Lymph Node Metastasis and Predicts Prognosis in Resectable Esophageal Cancer. World I Surg. 2017:41(8):2068-77.

24. Mei Y, Liu H, Sun X, Li X, Zhao S, Ma R. Plasma fibrinogen level may be a possible marker for the clinical response and prognosis of patients with breast cancer receiving neoadjuvant chemotherapy. Tumour Biol 2017;39(6): 1010428317700002.

25. Luo Y, Kim HS, Kim M, Lee M, Song YS. Elevated plasma fibrinogen levels and prognosis of epithelial ovarian cancer: a cohort study and metaanalysis. J Gynecol Oncol. 2017;28(3):e36.

26. Palumbo JS, Kombrinck KW, Drew AF, Grimes TS, Kiser JH, Degen JL, et al. Fibrinogen is an important determinant of the metastatic potential of circulating tumor cells. Blood. 2000;96(10):3302-9.

27. Perisanidis C, Psyrri A, Cohen EE, Engelmann J, Heinze G, Perisanidis B, et al. Prognostic role of pretreatment plasma fibrinogen in patients with solid tumors: A systematic review and meta-analysis. Cancer Treat Rev. 2015; 41(10):960-70.

\section{Publisher's Note}

Springer Nature remains neutral with regard to jurisdictional claims in published maps and institutional affiliations.

Ready to submit your research? Choose BMC and benefit from:

- fast, convenient online submission

- thorough peer review by experienced researchers in your field

- rapid publication on acceptance

- support for research data, including large and complex data types

- gold Open Access which fosters wider collaboration and increased citations

- maximum visibility for your research: over $100 \mathrm{M}$ website views per year

At BMC, research is always in progress.

Learn more biomedcentral.com/submissions 